\title{
A Lower Bound for the Class Number of Certain Cubic Number Fields
}

\author{
By Günter Lettl
}

Abstract. Let $K$ be a cyclic number field with generating polynomial

$$
X^{3}-\frac{a-3}{2} X^{2}-\frac{a+3}{2} X-1
$$

and conductor $m$. We will derive a lower bound for the class number of these fields and list all such fields with prime conductor $m=\left(a^{2}+27\right) / 4$ or $m=\left(1+27 b^{2}\right) / 4$ and small class number.

1. Introduction. Let $h_{m}$ denote the class number of the cyclotomic field $\mathbf{Q}\left(\zeta_{m}\right)$, and $h_{m}^{+}$, the class number of its maximal real subfield $\mathbf{Q}(\cos (2 \pi / m))$. It is a well-known conjecture of Vandiver that $p+h_{p}^{+}$holds for all primes $p \in \mathbf{P}$. This is a customary assumption for proving the second case of Fermat's Last Theorem (for more details see Washington [16]). Since $h_{p}^{+}$grows slowly $\left(h_{p}^{+}=1\right.$ for $p<163$ with the use of the Generalized Riemann Hypothesis (GRH), van der Linden [10]), for no $p$ with $h_{p}^{+}>1$ the exact value of $h_{p}^{+}$is known without using GRH. Masley suggested that perhaps $h_{p}^{+}<p$ always holds, but a counterexample was found in [3], [12]. The class number of each real subfield of $\mathbf{Q}\left(\zeta_{p}\right)$ divides $h_{p}^{+}$, and in this way one can find primes with $h_{p}^{+}>1$. Using the quadratic subfield, Ankeny, Chowla and Hasse [1] showed that $h_{p}^{+}>1$ if $p$ belongs to certain quadratic sequences in $\mathbf{N}$, and S.-D. Lang [9] and Takeuchi [15] found more such sequences. Similar results were obtained for $h_{4 p}^{+}$by Yokoi [17]. Using the cubic subfield of $\mathbf{Q}\left(\zeta_{p}\right)$, which has been thoroughly investigated (e.g., [2], [5], [8]), the theorem of the present paper yields the following results:

If $a$ is an odd integer, $a>23$, and $p=\left(a^{2}+27\right) / 4$, a prime, then $h_{p}^{+}>1$.

If $b$ is an odd integer, $b>7$, and $p=\left(1+27 b^{2}\right) / 4$, a prime, then $h_{p}^{+}>1$.

A conjecture about primes in quadratic sequences (Hardy and Wright [7, I.2.8]) implies that there exist infinitely many primes $p$ of each of these two forms, because one can write

$$
\frac{a^{2}+27}{4}=\left(\frac{a-3}{2}\right)^{2}+3\left(\frac{a-3}{2}\right)+9
$$

and

$$
\frac{1+27 b^{2}}{4}=3\left(\frac{3 b-1}{2}\right)^{2}+3\left(\frac{3 b-1}{2}\right)+1
$$

Received November 6, 1984; revised July 15, 1985

1980 Mathematics Subject Classification. Primary 12A30, 12A50.

(C)1986 American Mathematical Society $0025-5718 / 86 \$ 1.00+\$ .25$ per page 
2. Class Number Bounds and Main Results. Let $K$ be a cyclic cubic number field with conductor $m$ and class number $h$. It is well known that $m$ is the product of distinct primes, which are congruent to $1 \bmod (6)$, and of 9 , if 3 ramifies in $K$. The class number $h$ is congruent to $1 \bmod (3)$, if $m$ is a prime or $m=9$, and $h$ is divisible by 3 otherwise. Set $f(s)=L(s, \chi) \cdot L(s, \bar{\chi})$ for $s \in \mathbf{C}$, where $\chi$ and $\bar{\chi}$ are the nontrivial cubic Dirichlet characters modulo $(m)$ belonging to $K$. Since the discriminant of $K$ equals $m^{2}$, the analytic class number formula yields

$$
h=\frac{m \cdot f(1)}{4 \cdot R}
$$

where $R$ is the regulator of $K$. Moser [11] showed that for prime conductors, $h<m / 3$ holds, so cubic fields will never lead to a contradiction to Vandiver's conjecture. Our aim is to establish a lower bound for the class number of a special family of cubic fields and to list all fields of some special types with prime conductor and small class number. From a result of Stark [14] one can deduce $f(1)>c / \log m$, where $c$ is effectively computable, but this bound is not suited for our purposes. From the results of the next section we will obtain:

$$
\begin{aligned}
& \text { If } K \text { is a cyclic cubic number field with conductor } m>10^{5} \text {, then } \\
& f(1)>0.023 \cdot m^{-0.054} \text {. }
\end{aligned}
$$

The harder problem is to find an upper bound for the regulator, which is only achieved for the following family of cyclic cubic fields. The polynomial

$$
f_{a}(X)=X^{3}-\frac{a-3}{2} X^{2}-\frac{a+3}{2} X-1, \quad a \in \mathbf{N} \text { odd }
$$

is irreducible over $\mathbf{Q}$, has discriminant $D\left(f_{a}\right)=\left(\left(a^{2}+27\right) / 4\right)^{2}$, and if $\varepsilon$ is a zero of $f_{a}$, the other zeros are $\varepsilon^{\prime}=-1 /(\varepsilon+1)$ and $\varepsilon^{\prime \prime}=-(\varepsilon+1) / \varepsilon$. Therefore, $f_{a}$ is a generating polynomial of a cyclic cubic field $K$ with conductor $m$, and we define $k \in \mathbf{N}$ by $\sqrt{D\left(f_{a}\right)}=\left(a^{2}+27\right) / 4=k m$.

We call the field $K$ of type A, if $k=1$, and of type $\mathrm{B}$, if $k=27$ and $a=27 b$ with $b \in \mathbf{N}$ odd, $b \neq 1$ (in this case we have $\left.m=\left(1+27 b^{2}\right) / 4\right)$. It is well known that fields of type A or B have relatively large class numbers (see, for example, the tables of Gras [5]). Shanks [13] states that for cubic fields of type A with prime conductor "a rough mean value for $h$ is given by $h \approx 12 m / 35(\log m)^{2}$ ".

LEMMA 1. Let $K$ be a cyclic cubic field with generating polynomial $f_{a}$, conductor $m$ and regulator $R$. Then,

$$
4 R<\left(\frac{1}{2} \log D\left(f_{a}\right)\right)^{2}=(\log (k m))^{2} .
$$

Proof of Lemma 1. Since the zeros $\varepsilon, \varepsilon^{\prime}, \varepsilon^{\prime \prime}$ of $f_{a}$ are units of $K$, we can estimate the regulator of $K$ by $R \leqslant \operatorname{Reg}\left(\left\{\varepsilon, \varepsilon^{\prime}\right\}\right)=: R^{\prime}$, if $R^{\prime} \neq 0$ (see Lemma 4.15 in [16]). Choosing

$$
\varepsilon=\frac{a-3+4 \sqrt{k m} \cdot \cos (1 / 3 \cdot \arctan (\sqrt{27} / a))}{6} \sim \sqrt{k m}
$$


with the principal value of arctan, we obtain

$$
\begin{aligned}
R^{\prime} & =\left|\operatorname{det}\left(\begin{array}{cc}
\log |\varepsilon| & \log \left|\varepsilon^{\prime}\right| \\
\log \left|\varepsilon^{\prime}\right| & \log \left|\varepsilon^{\prime \prime}\right|
\end{array}\right)\right| \\
& =(\log |\varepsilon+1|)^{2}-\log |\varepsilon+1| \log |\varepsilon|+(\log |\varepsilon|)^{2} .
\end{aligned}
$$

Series expansions yield

$$
R^{\prime}=\frac{1}{4}(\log k m)^{2}-\frac{3 \log (k m)}{2 k m}+\frac{3}{4 k m}+O\left(\frac{\log k m}{(k m)^{2}}\right)
$$

and elementary calculus explicitly gives (4).

With (2) and Lemma 1 we immediately obtain from (1):

Let $K$ be a cyclic cubic field with conductor $m>10^{5}$ and with generating polynomial $f_{a}$. Then,

$$
h>0.023 \frac{m^{0.946}}{(\log k m)^{2}} .
$$

THEOREM. (a) Let $K$ be a cyclic cubic field of type A with prime conductor $m$. Then $h<16$ holds only for the following values of $m$ :

\begin{tabular}{r|l}
$h$ & \multicolumn{1}{|c}{$m$} \\
\hline 1 & $7,13,19,37,79,97,139$ \\
4 & $163,349,607,709,937$ \\
7 & $313,877,1129,1567,1987,2557$ \\
13 & 1063
\end{tabular}

(b) Let $K$ be a cyclic cubic field of type $\mathrm{B}$ with prime conductor $m$. Then $h<43$ holds only for the following values of $m$ :

\begin{tabular}{r|l}
$h$ & \multicolumn{1}{|c}{$m$} \\
\hline 1 & 61,331 \\
4 & 547,1951 \\
7 & 2437,3571 \\
13 & 9241 \\
28 & 4219,25117 \\
31 & 23497 \\
37 & 8269
\end{tabular}

Proof of the Theorem. From (5) we obtain $h>14$ for fields of type A with $m \geqslant 169339$, and $h>37.2$ for fields of type B with $m>10^{6}$. It is well known (see, e.g., Gras [4]) that primes $q \equiv-1 \bmod (3)$ divide the class number of a cyclic cubic field only with an even exponent. The table of class numbers of Shanks [13], and Table 1 below, complete the proof of the theorem. 
TABLE 1

Class numbers of cyclic cubic fields of type $\mathrm{B}$

with prime conductor $m<10^{6}$

\begin{tabular}{r|r|l}
\hline$b$ & $m=\frac{1+27 b^{2}}{4}$ & \multicolumn{1}{|c}{$h$} \\
\hline & & \\
3 & 61 & 1 \\
7 & 331 & 1 \\
9 & 547 & $4=2^{2}$ \\
17 & 1951 & $4=2^{2}$ \\
19 & 2437 & 7 \\
23 & 3571 & 7 \\
25 & 4219 & $28=2^{2} \cdot 7$ \\
33 & 7351 & $49=7^{2}$ \\
35 & 8269 & 37 \\
37 & 9241 & 13 \\
39 & 10267 & $49=7^{2}$ \\
45 & 13669 & 109 \\
59 & 23497 & 31 \\
61 & 25117 & $28=2^{2} \cdot 7$ \\
91 & 55897 & $133=7 \cdot 19$ \\
95 & 60919 & 193 \\
105 & 74419 & $688=2^{4} \cdot 43$ \\
115 & 89269 & 211 \\
117 & 92401 & $532 \quad 2^{2} \cdot 7 \cdot 19$ \\
123 & 102121 & 307 \\
129 & 112327 & $604=2^{2} \cdot 151$ \\
131 & 115837 & $148=2^{2} \cdot 37$ \\
137 & 126691 & 97 \\
147 & 145861 & $652=2^{2} \cdot 163$ \\
159 & 170647 & $628=2^{2} \cdot 157$ \\
\hline
\end{tabular}

\begin{tabular}{|c|c|c|}
\hline$b$ & $m=\frac{1+27 b^{2}}{4}$ & $h$ \\
\hline 173 & 202021 & $316=2^{2} \cdot 79$ \\
\hline 185 & 231019 & $343=7^{3}$ \\
\hline 189 & 241117 & $1216=2^{6} \cdot 19$ \\
\hline 191 & 246247 & $175=5^{2} \cdot 7$ \\
\hline 193 & 251431 & $247=13 \cdot 19$ \\
\hline 199 & 267307 & $196=2^{2} \cdot 7^{2}$ \\
\hline 205 & 283669 & 541 \\
\hline 221 & 329677 & $316=2^{2} \cdot 79$ \\
\hline 227 & 347821 & 331 \\
\hline 231 & 360187 & $1732=2^{2} \cdot 433$ \\
\hline 235 & 372769 & $553=7 \cdot 79$ \\
\hline 243 & 398581 & $1075=5^{2} \cdot 43$ \\
\hline 259 & 452797 & 769 \\
\hline 261 & 459817 & $2257=37 \cdot 61$ \\
\hline 297 & 595411 & $2299=11^{2} \cdot 19$ \\
\hline 299 & 603457 & 739 \\
\hline 301 & 611557 & $889=7 \cdot 127$ \\
\hline 303 & 619711 & $1156=2^{2} \cdot 17^{2}$ \\
\hline 305 & 627919 & $1552=2^{4} \cdot 97$ \\
\hline 341 & 784897 & $688=2^{4} \cdot 43$ \\
\hline 347 & 812761 & 769 \\
\hline 361 & 879667 & $688=2^{4} \cdot 43$ \\
\hline 367 & 909151 & 787 \\
\hline 371 & 929077 & $1588=2^{2} \cdot 397$ \\
\hline 373 & 939121 & 661 \\
\hline 383 & 990151 & $532=2^{2} \cdot 7 \cdot 19$ \\
\hline
\end{tabular}

The class numbers of Table 1 were calculated with a "Sirius 1 Personal Computer", using the analytic class number formula (1). We also used that for fields of type B the roots of $f_{a}$ are already fundamental units, and therefore $R=R^{\prime}$ can be calculated with the explicit formula for $\varepsilon$, given in the proof of Lemma 1 . In the following way it can be proved that $\varepsilon$ is a fundamental unit:

Let $K$ be a field of type $\mathrm{B}$ with generating polynomial $f_{a}, a=27 b$ and $m=\left(1+27 b^{2}\right) / 4$. Hasse [8] investigated the arithmetic of cyclic cubic fields, using the Gauss sums of the corresponding Dirichlet characters. With Hasse's notation, every integer $\alpha \in K$ can be written as $\alpha=[x, y]$ with $x \in \mathbf{Z}, y \in \mathbf{Z}[\rho]$, where $\rho^{2}+\rho+1=0$, and $x \equiv y \bmod (1-\rho)$. If $\alpha$ is a unit of $K, N(\alpha)=1$ implies $x^{3} \equiv 27 \bmod (m)$ and $|x| \leqslant 2 \sqrt{m y \bar{y}}$ (Satz 8, [8]). For the roots of $f_{27 b}$ we have $\varepsilon=[(27 b-3) / 2,3 i \sqrt{3}]$ and its conjugates. Since Godwin's conjecture about fundamental units holds for cyclic cubic fields with $m>9$ (see Gras [6]), we have to show:

There exists no unit $\alpha=[x, y] \in K, \alpha \neq \pm 1$, with $m y \bar{y}=\frac{1}{2} \operatorname{tr}\left(\alpha-\alpha^{\prime}\right)^{2}$ $<\frac{1}{2} \operatorname{tr}\left(\varepsilon-\varepsilon^{\prime}\right)^{2}=27 m$, where $\operatorname{tr}$ denotes the trace from $K$ to $\mathbf{Q}$.

Suppose the contrary. Then $x^{3} \equiv 27 \bmod (m)$ and $|x|<2 \sqrt{27 m}$ imply $x \in$ $\{3,(27 b-3) / 2,-(27 b+3) / 2\}$ for $b \geqslant 7$. Considering $0 \equiv x \equiv y \bmod (1-\rho)$ and $y \bar{y}<27$ yields only a few possibilities for $y \in \mathbf{Z}[\rho]$, and one can check that for each 
of these $y, N(\alpha)=1$ has no solution $\alpha \neq 1$. For small values of $b$, one can consult the table in [5].

In the same way, but with much less computation, one can prove that for $k=1$ (type A) and $k=3$ the roots of $f_{a}$ are also fundamental units. In these cases one has $\varepsilon=[(a-3) / 2, \pm 1]$ with $(a-3) / 2 \equiv \pm 1 \bmod (3)$, and $\varepsilon=[(9 b-3) / 2, i \sqrt{3}]$, respectively.

3. A Lower Bound for $L(1, \chi) \cdot L(1, \bar{\chi})$. Let $m$ be the conductor of a cyclic cubic field $K, \chi$ and $\bar{\chi}$ the nontrivial cubic Dirichlet characters modulo $m$ associated with $K$, and $f(s)=L(s, \chi) L(s, \bar{\chi})$. To find a lower bound for $f(1)$, we first need an upper bound for $|f(s)|$ in a disk in $\mathbf{C}$ containing 1 . Consider $C=C(\mu, \rho)=$ $\{s \in \mathbf{C}|| s-\mu \mid<\rho\}$ with $1<\mu$ and $\mu-1<\rho<\mu$, and set $\sigma_{0}=\mu-\rho$. Let $s=$ $\boldsymbol{\sigma}+i t \in \mathbf{C}$. For $\boldsymbol{\sigma}>0$ we have the representation

$$
L(s, \chi)=\sum_{n=1}^{m-1} \frac{\chi(n)}{n^{s}}+s \cdot \int_{m}^{\infty} \frac{S(x, \chi)}{x^{s+1}} d x \quad \text { with } S(x, \chi)=\sum_{1 \leqslant n<x} \chi(n)
$$

(see [16, p. 211]). The inequality of Pólya-Vinogradov [16, Lemma 11.8] states that $|S(x, \chi)|<\sqrt{m} \cdot \log m$. For $s \in C(\mu, \rho)$, the function $|s| / \sigma=1 / \cos (\arg s)$ attains its maximum $\mu / \sqrt{\mu^{2}-\rho^{2}}$ if $s$ is the point of contact of a tangent of $C$ through 0 . Combining these results, we obtain for every $s \in C(\mu, \rho)$ :

$$
\begin{aligned}
|L(s, \chi)| & <1+\int_{1}^{m} \frac{1}{x^{\sigma_{0}}} d x+|s| \sqrt{m} \cdot \log m \int_{m}^{\infty} \frac{1}{x^{\sigma+1}} d x \\
& <\frac{1}{1-\sigma_{0}} m^{1-\sigma_{0}}+\frac{\mu}{\sqrt{\mu^{2}-\rho^{2}}} \log m \cdot m^{0.5-\sigma_{0}} .
\end{aligned}
$$

Since $\log x / \sqrt{x}$ is monotone decreasing for $x \geqslant e^{2}$, we conclude that for $m \geqslant m_{0} \geqslant$ $e^{2}$

$$
|f(s)|<c_{1} \cdot m^{2-2 \sigma_{0}}
$$

holds for all $s \in C(\mu, \rho)$, with

$$
c_{1}=\left(\frac{1}{1-\sigma_{0}}+\frac{\mu}{\sqrt{\mu^{2}-\rho^{2}}} \cdot \frac{\log m_{0}}{\sqrt{m_{0}}}\right)^{2} .
$$

LEMMA 2. If $K$ is a cyclic cubic number field with conductor $m$, then $f(1)>c_{6} \cdot m^{-c_{7}}$, with $c_{6}, c_{7}>0$ as given in the course of the proof. Furthermore, $c_{7}$ can be made arbitrarily small.

Proof of Lemma 2. The proof follows mainly Washington [16, pp. 212-214]. Let $\zeta(s)$ be the Riemann zeta function and $\zeta_{K}(s)=\zeta(s) f(s)$ the zeta function of the cyclic cubic field $K$ with conductor $m$. If $s=\sigma+i t \in \mathbf{C}$, we have

$$
\zeta_{K}(s)=1+\sum_{n=2}^{\infty} \frac{a_{n}}{n^{s}} \text { for } \sigma>1
$$

with $a_{n} \geqslant 0$, and $a_{n} \geqslant 1$ if $n$ is a cube. Developing $\zeta_{K}$ in a power series around $\mu>1$ gives

$$
\zeta_{K}(s)=\sum_{j=0}^{\infty} b_{j}(\mu-s)^{j}
$$


with

(7) $b_{0}=\zeta_{K}(\mu)>\zeta(3 \mu)>1$ and $b_{j}=\frac{1}{j !} \sum_{n=2}^{\infty}(\log n)^{j} \cdot \frac{a_{n}}{n^{\mu}}>0$ for $j \geqslant 1$.

The integral representation of $\zeta(s)$ for $\sigma>0$ yields

$$
|\zeta(s)| \leqslant\left|\frac{s}{s-1}\right|+|s| \int_{1}^{\infty} \frac{1}{u^{\sigma+1}} d u=\left|\frac{s}{s-1}\right|+\frac{|s|}{\sigma}
$$

and

$$
\begin{aligned}
|\zeta(s)| & \leqslant\left|\frac{s}{s-1}\right|+|s| \cdot \sum_{n=1}^{\infty} \frac{1}{n^{\sigma+1}} \cdot \int_{n}^{n+1}(u-[u]) d u \\
& <\left|\frac{s}{s-1}\right|+\frac{|s|}{2}\left(1+\frac{1}{\sigma}\right) .
\end{aligned}
$$

Let $C=C(\mu, \rho)$, with $\mu-1<\rho<\mu$, be the disk with center $\mu$ and radius $\rho$, and denote its boundary by $\partial C$. Using (6), we get for all $s \in \partial C$ :

$$
\left|\zeta_{K}(s)-\frac{f(1)}{s-1}\right| \leqslant|\zeta(s)| \cdot|f(s)|+\frac{1}{|s-1|} \cdot|f(1)|<c_{2} \cdot m^{2-2 \sigma_{0}}
$$

with

$$
c_{2}=c_{1} \cdot \max _{s \in \partial C}\left(\frac{|s|+1}{|s-1|}+|s| \cdot \min \left\{\frac{1}{\sigma}, \frac{1}{2}\left(1+\frac{1}{\sigma}\right)\right\}\right) .
$$

Since $\zeta_{K}(s)-f(1) /(s-1)$ is holomorphic in the whole complex plane, (8) holds for all $s \in C(\mu, \rho)$. Computing the coefficients of

$$
\zeta_{K}(s)-\frac{f(1)}{s-1}=\sum_{j=0}^{\infty}\left(b_{j}-\frac{f(1)}{(\mu-1)^{j+1}}\right) \cdot(\mu-s)^{j}
$$

with a Cauchy integral gives

$$
\left|b_{j}-\frac{f(1)}{(\mu-1)^{j+1}}\right|=\left|\frac{1}{2 \pi i} \int_{\partial C}\left(\zeta_{K}(s)-\frac{f(1)}{s-1}\right) \frac{d s}{(s-\mu)^{j+1}}\right|<\frac{c_{2}}{\rho^{j}} \cdot m^{2-2 \sigma_{0}} .
$$

For $0<\sigma<1$, the integral representation of $\zeta(s)$, and $f(\sigma)=|L(\sigma, \chi)|^{2}$, show that $\zeta_{K}(\sigma) \leqslant 0$. So for any $\alpha$ with $\sigma_{0}<\alpha<1$, and any $\nu \in \mathbf{R}^{+}$with $1<\nu$, we have

$$
\begin{aligned}
&-\frac{f(1)}{\alpha-1} \geqslant \zeta_{K}(\alpha)-\frac{f(1)}{\alpha-1}>\sum_{j=0}^{[\nu]-1}\left(b_{j}-\frac{f(1)}{(\mu-1)^{j+1}}\right) \cdot(\mu-\alpha)^{j} \\
&-c_{2} \cdot m^{2-2 \sigma_{0}} \cdot \sum_{j=[\nu]}^{\infty}\left(\frac{\mu-\alpha}{\rho}\right)^{j} \\
& \geqslant c_{3}-\frac{f(1)}{\alpha-1}-\frac{f(1)}{1-\alpha}\left(\frac{\mu-\alpha}{\mu-1}\right)^{\nu}-c_{2} \cdot m^{2-2 \sigma_{0}}\left(\frac{\mu-\alpha}{\rho}\right)^{\nu-1} \cdot \frac{\rho}{\alpha-\sigma_{0}}, \\
& \text { where } \sum_{j=0}^{[\nu]-1} b_{j}(\mu-\alpha)^{j} \geqslant c_{3} \geqslant 1 .
\end{aligned}
$$

From this inequality, we obtain

$$
f(1)>c_{3}(1-\alpha)\left(\frac{\mu-1}{\mu-\alpha}\right)^{\nu}-c_{2} \cdot m^{2-2 \sigma_{0}} \cdot \frac{\rho^{2}(1-\alpha)}{(\mu-\alpha)\left(\alpha-\sigma_{0}\right)}\left(\frac{\mu-1}{\rho}\right)^{\nu} .
$$


Choosing $\nu=c_{4} \cdot \log m+c_{5}$, with

$$
c_{4}=\frac{2-2 \sigma_{0}}{\log \frac{\rho}{\mu-\alpha}}
$$

and

$$
c_{5}=\frac{\log \frac{c_{2} \cdot \rho^{2}}{(\mu-\alpha)\left(\alpha-\sigma_{0}\right)}+\log \log \frac{\rho}{\mu-1}-\log \log \frac{\mu-\alpha}{\mu-1}}{\log \frac{\rho}{\mu-\alpha}},
$$

gives $f(1)>c_{6} \cdot m^{-c_{7}}$, with

$$
c_{6}=c_{3}(1-\alpha)\left(\frac{\mu-1}{\mu-\alpha}\right)^{c_{s}}-c_{2} \cdot \frac{\rho^{2}(1-\alpha)}{(\mu-\alpha)\left(\alpha-\sigma_{0}\right)} \cdot\left(\frac{\mu-1}{\rho}\right)^{c_{s}}
$$

and

$$
c_{7}=\left(2-2 \sigma_{0}\right) \cdot \log \frac{\mu-\alpha}{\mu-1} / \log \frac{\rho}{\mu-\alpha} .
$$

Since $c_{7} \rightarrow 0$ for $\alpha \rightarrow 1$, the proof of Lemma 2 is completed.

Numerical computations show that for $m_{0}=10^{5}$ good results can be obtained by choosing $\mu=10, \rho=9.9$ and $\alpha=0.975$. With these values we obtain $c_{2}=10.8685$ and $\nu \approx 315$.

Using (7), and $a_{n} \geqslant 1$ for $n$ a cube, we obtain the following estimations for $c_{3}$ :

$$
\begin{aligned}
& \sum_{j=0}^{[\nu]-1} b_{j}(\mu-\alpha)^{j} \geqslant \zeta_{K}(\mu)+\sum_{j=1}^{300} \frac{1}{j !} \sum_{n=2}^{\infty} \frac{a_{n}}{n^{\mu}}((\mu-\alpha) \log n)^{j} \\
& \quad>\zeta(3 \mu)+\sum_{k=2}^{N_{0}} \frac{1}{k^{3 \mu}} \sum_{j=1}^{300} \frac{1}{j !}((\mu-\alpha) 3 \cdot \log k)^{j} \\
& \quad>1+\sum_{k=2}^{N_{0}} \frac{1}{k^{3 \mu}}\left(k^{3(\mu-\alpha)}-\frac{((\mu-\alpha) 3 \cdot \log k)^{301}}{301 !} \cdot \frac{302}{302-(\mu-\alpha) 3 \cdot \log k}\right)
\end{aligned}
$$

where $N_{0}<e^{302 / 3(\mu-\alpha)}$. With the special values for $\mu, \rho$ and $\alpha$, and $N_{0}=100$, we obtain

$$
\sum_{j=0}^{[\nu]-1} b_{j}(\mu-\alpha)^{j}>\sum_{k=1}^{100} k^{-2.925}-10^{-40}>1.2175=c_{3} .
$$

These values yield $c_{6}>0.023$ and $c_{7}<0.054$, and thus (2) is proved.

Institut für Mathematik

Karl-Franzens-Universität

Halbärthgasse 1

A-8010 Graz

Österreich, Austria

1. N. C. Ankeny, S. Chowla \& H. Hasse, “On the class-number of the maximal real subfield of a cyclotomic field," J. Reine Angew. Math., v. 217, 1965, pp. 217-220.

2. A. ChÂtelet, "Arithmétique des corps abéliens du troisième degré," Ann. Sci. École Norm. Sup. (3), v. 63, 1946, pp. 109-160.

3. G. Cornell \& L. C. Washington, “Class numbers of cyclotomic fields,” J. Number Theory, v. 21, 1985 , pp. 260-274. 
4. M.-N. Gras, Sur le Nombre de Classes du Sous-Corps Cubique de $\mathbf{Q}^{(p)}(p \equiv 1$ (3)), Thèse, Grenoble, 1971.

5. M.-N. Gras, "Méthodes et algorithmes pour le calcul numérique du nombre de classes et des unités des extensions cubiques cycliques de Q," J. Reine Angew. Math., v. 277, 1975, pp. 89-116.

6. M.-N. Gras, "Note a propos d'une conjecture de H. J. Godwin sur les unités des corps cubiques," Ann. Inst. Fourier (Grenoble), v. 30/4, 1980, pp. 1-6.

7. G. H. Hardy \& E. M. Wright, An Introduction to the Theory of Numbers, 5th ed., Oxford Univ. Press, New York, 1979.

8. H. HASSE, "Arithmetische Bestimmung von Grundeinheit und Klassenzahl in zyklischen kubischen und biquadratischen Zahlkörpern," Abh. Deutsch. Akad. Wiss. Berlin Math.-Nat. Kl., 1948, Nr. 2, 95 pp.

9. S.-D. LANG, "Note on the class-number of the maximal real subfield of a cyclotomic field", $J$. Reine Angew. Math., v. 290, 1977, pp. 70-72.

10. F. J. VAN DER Linden, "Class number computations of real abelian number fields," Math. Comp., v. 39, 1982, pp. 693-707.

11. C. Moser, Sur le Nombre de Classes d'un Corps $K$ Réel Cyclique de Conducteur Premier, deg $K \geqslant 4$, Seminaire Théorie des Nombres, Grenoble, 1980, 17 pp.

12. E. Seah, L. C. Washington \& H. C. Williams, "The calculation of a large cubic class number with an application to real cyclotomic fields", Math. Comp., v. 41, 1983, pp. 303-305.

13. D. SHanKs, “The simplest cubic fields," Math. Comp., v. 28, 1974, pp. 1137-1152.

14. H. M. STARK, "Some effective cases of the Brauer-Siegel Theorem," Invent. Math., v. 23, 1974, pp. 135-152.

15. H. TAKEUCHI, "On the class-number of the maximal real subfield of a cyclotomic field," Canad. J. Math., v. 33, 1981, pp. 55-58.

16. L. C. WASHIngton, Introduction to Cyclotomic Fields, GTM 83, Springer, New York, 1982.

17. H. YoKol, "On the diophantine equation $x^{2}-p y^{2}= \pm 4 q$ and the class number of real subfields of a cyclotomic field," Nagoya Math. J., v. 91, 1983, pp. 151-161. 